\title{
Nanoflow liquid chromatography coupled to matrix- assisted laser desorption/ionization mass spectrometry: Sample preparation, data analysis, and application to the analysis of complex peptide mixtures
}

\author{
Ekaterina Mirgorodskaya, Corina Braeuer, Paola Fucini, Hans Lehrach and Johan Gobom \\ Max Planck Institute for Molecular Genetics, Berlin, Germany
}

\begin{abstract}
We report the development of a robust interface for off-line coupling of nano liquid chromatography (LC) to matrix-assisted laser desorption/ionisation-mass spectrometry (MALDI-MS) and its application to the analysis of proteolytic digests of proteins, both isolated and in mixtures. The interface makes use of prestructured MALDI sample supports to concentrate the effluent to a small sample plate area and localize the MALDI sample to a predefined array, thereby enriching the analyte molecules and facilitating automated MALDI-MS analysis. Parameters that influence the preparation of MALDI samples from the LC effluent were evaluated with regard to detection sensitivity, spectra quality, and reproducibility of the method. A procedure for data processing is described. The presented nano LC MALDI-MS system allowed the detection of several peptides from a tryptic digest of bovine serum albumin, at analyzed amounts corresponding to one femtomole of the digested protein. For the identification of native proteins isolated from mouse brain by two-dimensional gel electrophoresis, nano LC MALDI-MS increased the number of detected peptides, thereby allowing identification of proteins that could not be identified by direct MALDIMS analysis. The ability to identify proteins in complex mixtures was evaluated for the analysis of Escherichia coli $50 \mathrm{~S}$ ribosomal subunit. Out of the 33 expected proteins, 30 were identified by MALDI tandem time of flight fragment ion fingerprinting.
\end{abstract}

\section{Keywords:}

Liquid chromatography, Matrix-assisted laser desorption/ionisation / Peptides / Protein identification / Tandem mass spectrometry

\section{Introduction}

Liquid chromatography at flow rates in the nanoliter/minute range (nano LC) coupled to MS is often used for the analysis of peptide mixtures derived from biological sources. The small surface areas and dead-volumes of nano LC systems minimize analyte losses due to surface adsorption, and the low flow rates reduce analyte dilution. Thereby, analytes of low abundance can be separated with a high recovery.

Correspondence: Dr. Ekaterina Mirgorodskaya, Max-Planck Institute for Molecular Genetics, Ihnestrasse 73, D-14195 Berlin, Germany

E-mail: mirgorod@molgen.mpg.de

Fax: +49-30-84131139
To date, nano LC-MS has mainly been used with electrospray mass spectrometry (ESI-MS) as detector of the separated analytes (see review [1, 2]). The ESI ion source requires a continuous supply of analytes in the liquid phase and is therefore naturally suited for on-line coupling to liquid separations. For separation of peptides, reversedphase (RP) chromatography is the most frequently used separation mode. Operating with water/organic solvent mixtures, RP chromatography allows direct infusion of the chromatographic effluent, or fractions thereof, into the ES ion source.

MALDI [3], in contrast, is a pulsed ionization technique, and requires the sample to be introduced in a condensed state, mixed with an active matrix compound. The matrices most commonly used for peptide analysis, such as CHCA, 
are solid compounds and the cocrystallization of analyte and matrix is an important part of the sample preparation. Using MALDI-MS as a downstream detector for liquid separations is therefore more readily performed off-line, by continuous or discrete deposition of LC effluent onto the MALDI sample support [4].

The off-line nature of the MALDI interface offers some distinct advantages compared to ESI for the analysis of chromatographic effluent. With LC-ESI MS, the time available for analysis of a compound is limited to the time-width of its chromatographic peak. In complex peptide mixtures, in which several analytes co-elute, this time interval is often too short for acquisition of fragment ion spectra of all sample components. This time constraint does not apply to MALDIMS. After initial mass analysis, which only consumes a minor part of the sample, the remainder of the sample can be stored on the MALDI sample plate for at least several days without degradation of the analyte molecules. This allows use of a different data acquisition strategy: first, all LC fractions are analyzed in MS mode, then the information derived from the peptide molecular masses in all fractions is used to determine which analytes are of interest for MS/MS analysis. Similarly, the information obtained by a first set of MS/MS analyses can be used to guide the selection of precursor ions for the following MS/MS analyses. In this way, LC-MALDI enables efficient data-dependent analyses.

Recent years have seen the development of a new generation of MALDI mass spectrometers with advanced MS/ MS capabilities, high spectra acquisition rates, instrument hardware and control software that allow fully automatic measurements $[5,6]$. This development has fulfilled the technical requirements for using MALDI as an attractive and efficient alternative to ESI for LC-MS/MS of complex peptide mixtures.

An important aspect of LC-MALDI is the design of the interface that mediates the transfer of the LC effluent to the MALDI sample support (see review [4]). For off-line interfaces, several different techniques for deposition of discrete LC fractions onto the MALDI sample support have been described, including piezo-dispensing [7] and pulsed electric field transfer [8]. Also, interfaces that allow continuous deposition have been reported $[9,10]$. Alternatively, on-line interfaces have been developed by several groups [11-13].

Here we report on a robust interface developed in our laboratory for off-line coupling of nano LC to MALDI-MS and its performance for protein/peptide analysis. The interface makes use of prestructured MALDI sample supports to concentrate the effluent to sample spots with a diameter of $600 \mu \mathrm{m}$, thereby enhancing detection sensitivity. By localizing the samples to a predefined array, automatic data acquisition is simplified. We evaluated several different methods and experimental parameters for the fractionation and MALDI sample preparation procedure. These included: assisting fractionation by a liquid sheath-flow, assisting the solvent evaporation by a gas sheath-flow, as well as optimization of the MALDI matrix solution composition.
The detection sensitivity of the method was evaluated, both by analyzing a known amount of peptides derived from tryptic cleavage of bovine serum albumin (BSA), and by comparing nano LC MALDI-TOF MS with direct MALDITOF MS mixture analysis of tryptic digests of mouse brain proteins isolated by 2-DE. The ability to use nano LC MALDIMS for identification of proteins in mixtures was evaluated for the analysis of the 50S ribosomal subunit from Escherichia coli, resulting in the successful identification of 30 ribosomal proteins out of the 33 expected.

\section{Materials and methods}

\subsection{Chemicals}

The peptide calibration standards, angiotensin I and ACTH 18-39 were purchased from Bachem (Heidelberg, Germany). Acetonitrile (HPLC gradient grade) was purchased from Carl Roth (Karlsruhe, Germany). TFA, tetrahydrofuran (THF), n-octylglucopyranoside (nOGP), CHCA, and water used for HPLC solvents and MALDI matrix solutions were purchased from Fluka (Buchs, Switzerland). Porcine trypsin was purchased from Promega (Mannheim, Germany), DTT, iodoacetamide (IAA), and bovine serum albumin (BSA) and citric acid from Sigma-Aldrich (St. Louis, MO, USA).

\subsection{Proteolysis of protein samples}

50S ribosomal subunits from E. coli were isolated by sucrose gradient centrifugation as previously described [14]. The concentration of $50 \mathrm{~S}$ subunits was determined by $\mathrm{UV}^{260}$ absorbance, where one unit corresponds to $36 \mathrm{pmol} / \mathrm{mL}$ of $50 \mathrm{~S} \mathrm{sub-}$ units [15]. A lyophilized sample containing 144 pmol (total protein amount) was dissolved in $50 \mathrm{~mm} \mathrm{NH} \mathrm{NCO}_{3}, 20 \mathrm{~mm}$ nOGP, pH $7.8(50 \mu \mathrm{L})$. For reduction of cysteines, a $5 \mu \mathrm{L}$ aliquot of $45 \mathrm{~mm}$ DTT was added and the sample was incubated at $37^{\circ} \mathrm{C}$ for $30 \mathrm{~min}$. Cysteine carbamidomethylation was performed by adding a $5 \mu \mathrm{L}$ aliquot of $100 \mathrm{~mm}$ IAA and incubating the sample at room temperature for $30 \mathrm{~min}$. The sample was diluted to $200 \mu \mathrm{L}$ by addition of $140 \mu \mathrm{L} \mathrm{H} \mathrm{H}_{2} \mathrm{O}$. A $10 \mu \mathrm{L}$ aliquot of trypsin $(50 \mathrm{ng} / \mu \mathrm{L})$ in $0.001 \% \mathrm{HCl} \mathrm{v} / \mathrm{v}$ was added and the sample was incubated at $37^{\circ} \mathrm{C}$ overnight. The sample was acidified and diluted by addition of $660 \mu \mathrm{L} 0.1 \%$ TFA v/v. A $2 \mu \mathrm{L}$ sample aliquot, corresponding to $330 \mathrm{fmol}$ of the purified protein complex, was used for analysis by nano LC MALDIMS. The same procedure for reduction, carbamidomethylation, and tryptic digestion was used for BSA. The mouse brain proteins isolated by 2-DE were subjected to in situ proteolysis as previously described [16], with minor modifications.

\subsection{Liquid chromatography}

Two different nano LC instruments were used in this study: an UltiMate ${ }^{\mathrm{TM}}$ nano HPLC system (LC Packings, Amsterdam, Netherlands) and an 1100 Series Nanoflow LC system 
(Agilent Technologies, Waldbronn, Germany). The mobile phases used for the RP separation were Buffer A: 1\% acetonitrile v/v, 0.05\% TFA v/v and Buffer B: $90 \%$ acetonitrile $\mathrm{v} / \mathrm{v}, 0.04 \%$ TFA v/v. Based on the analysis of a dilution series of a tryptic digest of BSA (see Section 3), the optimal sample load was determined to be in the range of 50 $100 \mathrm{fmol}$ of a digested protein. Typical sample loading volumes were $1-10 \mu \mathrm{L}$.

All initial nano LC MALDI experiments were performed using the UltiMate ${ }^{T M}$ nano LC system, consisting of a micro autosampler (FAMOS), a column switching module with a loading pump (Switchos), and a module containing a micropump and a UV detector (UltiMate). The samples were loaded onto a RP trapping column (PepMap ${ }^{\text {TM }} \mathrm{C}_{18}$, $300 \mu \mathrm{m} \times 5 \mathrm{~mm}$ ) using the loading pump operated at $20 \mu \mathrm{L} /$ min of Buffer A. After $8 \mathrm{~min}$, the 10-port valve was switched and the sample was eluted onto the analytical separation column (PepMap ${ }^{\mathrm{TM}} \mathrm{C}_{18}, 75 \mu \mathrm{m} \times 150 \mathrm{~mm}$ ) in back-flush mode using the micropump operated at $200 \mathrm{~nL} / \mathrm{min}$. The binary gradients, delivered by the micropump, were $1.5 \%$ Buffer B per min for tryptic digests of a single protein and 1\% Buffer B per min for the tryptic digest of the $50 \mathrm{~S}$ ribosomal subunit. Start and stop trigger signals for the xyz-stage were set at defined time points within the gradient, using external transistor-transistor logic (TTL) signals.

Alternatively, an 1100 Series Nanoflow LC was used. This system consists of a microwell plate autosampler, a binary loading pump, a thermostated column compartment with a micro 6-port/2-position valve, and a nanoflow pump equipped with an electronic flow control unit. The 1100 Series LC system was operated essentially as described for the UltiMate LC system. The samples were first loaded onto a trapping column (Zorbax $300 \mathrm{SB} \mathrm{C}_{18}, 0.3 \mathrm{~mm} \times 5 \mathrm{~mm}$ ), using Buffer A, delivered by the loading pump with a flow gradient according to the manufacturer's recommendation, i.e., $0.00 \mathrm{~min}-10 \mu \mathrm{L} / \mathrm{min}, 0.50 \mathrm{~min}-50 \mu \mathrm{L} / \mathrm{min}, 0.51 \mathrm{~min}-$ $10 \mu \mathrm{L} / \mathrm{min}$. After $10 \mathrm{~min}$, the trapping column was connected to the nanoflow path and the samples were eluted onto the analytical separation column (Zorbax $300 \mathrm{SB} \mathrm{C}_{18}$, $75 \mu \mathrm{m} \times 150 \mathrm{~mm}$ ), using a pump operated at $300 \mathrm{~nL} / \mathrm{min}$. A binary gradient of $1.5 \%$ Buffer B per min was used for separation of tryptic digests of isolated proteins. In addition, the use of flow-rate gradients from 300-500 nL/min during the analytical separation was tested for improving the robustness of the nano LC effluent fraction deposition onto the MALDI sample support. Two external contacts were used to trigger the xyz-stage at defined time points within the gradient.

\subsection{LC-MALDI sample preparation}

Prior to nano LC analysis, thin microcrystalline layers of CHCA were prepared on prestructured MALDI sample supports (AnchorChip $^{\text {TM }}$ 600/384; Bruker Daltonics, Bremen, Germany) according to a previously described procedure [17] with modifications made to the MALDI matrix solution. In brief, a $200 \mu \mathrm{L}$ aliquot of the matrix solution $(100 \mathrm{~g} / \mathrm{L}$ CHCA in $90 \%$ tetrahydrofuran, $0.001 \% \mathrm{TFA} \mathrm{v} / \mathrm{v}, 50 \mathrm{~mm}$ citric acid), containing the two calibration standards angiotensin I (1 pmol/ $\mu \mathrm{L})$ and ACTH $18-39(2 \mathrm{pmol} / \mu \mathrm{L})$, was deposited near one of the short edges of the support. A Teflon rod with $1 \mathrm{~mm}$ spacers at the ends was then placed parallel to the sample plate surface, contacting the matrix solution, which thereby spreads out along the rod. The rod was then drawn across the sample support, spreading the matrix solution. By this procedure, each hydrophilic anchor adsorbs a small volume of matrix solution, resulting after solvent evaporation, in uniform thin layers of crystalline CHCA in an ordered array.

An xyz-axis motion system (LND1; Luigs and Neumann, Ratingen, Germany) was used as the platform for the nano LC MALDI interface. The fused silica capillary carrying the effluent from the nano LC was threaded through a T-connection mounted on the axis system (Fig. 1a and b). The capillary outlet exits through a steel capillary, fitted with a thin polypropylene sleeve, mounted at the bottom of the Tconnection (Fig. 1b). Nitrogen (50 mbar), supplied through the left side of the T-connection, provided a gas sheath-flow around the emerging droplet of LC effluent, thereby assisting solvent evaporation and preventing analyte oxidation. The control software, written in LabView 6.0 (National Instruments, Munich, Germany), allows choice of different MALDI sample support geometries and control of the fraction deposition time. The positions of the first and the last fractions of each LC separation are recorded by the software. To ensure precise positioning of the capillary outlet above the MALDI sample support, required for low-volume fractionation, a three-point xyz-teaching defining the plane of the sample support was implemented. The optimal height of the capillary outlet above the sample support surface varied depending on the outer diameter (od) of the capillary. For fused silica capillaries of $280 \mu \mathrm{m}$ od, the height of the capillary outlet was set to $40 \pm 5 \mu \mathrm{m}$ above the surface, whereas, when using fused silica capillaries of $375 \mu \mathrm{m}$ od, the height of the capillary outlet was less critical and was set to $140 \pm 10 \mu \mathrm{m}$ above the surface. At the time-point corresponding to $15 \%$ Buffer B, a start trigger signal from the nano LC system initiates fraction collection. At time intervals defined by the user, typically $15 \mathrm{~s}$, the capillary was moved to a new sample plate position. Following the stop trigger signal from the LC system at 95\% Buffer B, the capillary was returned to the park position, at which it was positioned over (but not in direct contact with) a tissue paper to absorb the emerging LC effluent, while the LC system was reconditioned for the next run. Depending on the LC gradient, 90-150 fractions were collected from each separation. Prior to MS analysis, the MALDI samples were washed using an automatic liquid handler (TEMO; Tecan, Crailsheim, Germany) by adding aliquots of $3 \mu \mathrm{L}$ of $0.1 \%$ TFA to each sample. Following incubation for $3 \mathrm{~s}$ most of the liquid was removed by aspiration, and the small remainder was left to evaporate. 


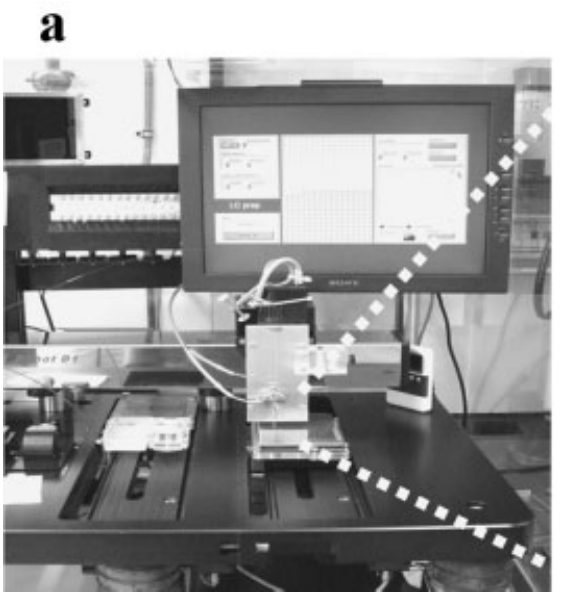

b

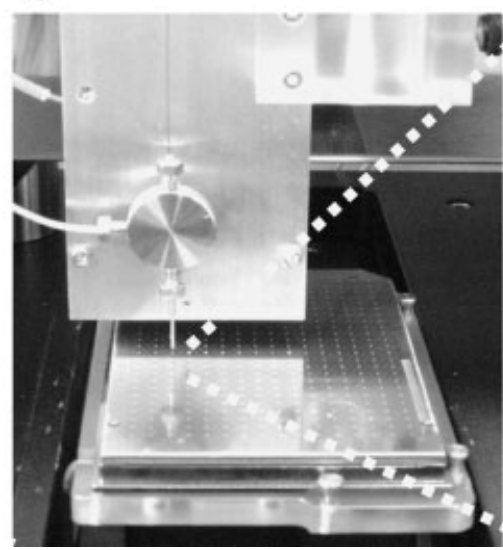

c

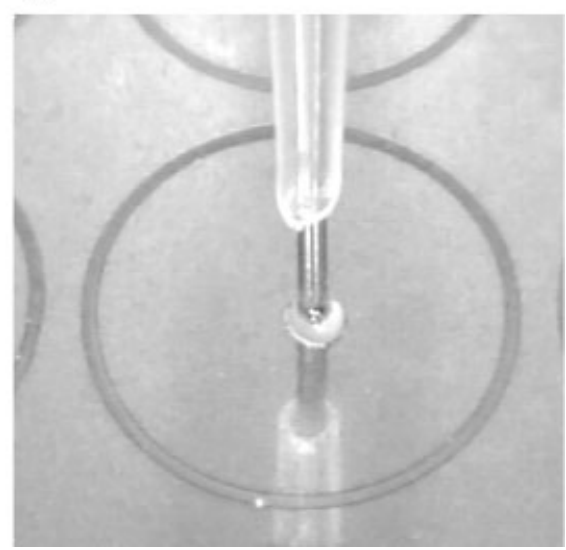

Figure 1. Experimental setup of the nano LC MALDI interface used in this study. (a) Overview of the xyz-axis motion system used for LC fraction collection onto MALDI sample supports. The fused silica capillary carrying the LC effluent is threaded through a T-connection mounted on the axis system. (b) Magnification of the T-connection. The capillary exits at the bottom of the T-connection through a stainless steel capillary, through which it protrudes a few millimeters. A stream of nitrogen gas, supplied through the left-hand port of the T-connection, exits in the space between the steel capillary and the fused silica LC capillary and provides a sheath-flow around the emerging LC effluent. (c) Magnification of the capillary outlet. During fractionation the capillary is positioned $40 \mu \mathrm{m}$ above the hydrophilic sample anchors (600 $\mu \mathrm{m}$ in diameter) of prestructured MALDI sample supports, prepared with thin layers of CHCA MALDI matrix.

\subsection{MALDI-TOF MS}

Mass analysis of positively charged peptide ions was performed on an Ultraflex LIFT and a Reflex III MALDI-TOF mass spectrometer (Bruker Daltonics). Positively charged ions in the mass range 500-4500 Da were analyzed automatically in the reflector mode. Sums of 30 single-shot spectra were acquired from ten different sample spot positions (300 in total from each sample). Fixed laser attenuation was used, the optimal value of which was determined prior to analysis by evaluation of a few fractions. MALDI-TOF/TOF analysis was performed on the Ultraflex instrument operated in LIFT mode, by operator-controlled data acquisition. Automatic detection of the peptide monoisotopic signals was performed using the algorithm SNAP, implemented in the FlexAnalysis software (Bruker Daltonics).

\subsection{Data processing}

The spectra were calibrated externally using a previously described procedure based on a polynomial function [18]. Internal mass correction was performed using the signals of two peptides (Angiotensin I, $\mathrm{MH}^{+}$(monoisotopic): 1,296.6853, and ACTH (18-39), $\left.\mathrm{MH}^{+} 2,465.1989\right)$ included in the MALDI matrix solution, as reference masses. Filtering of background signals, grouping of signals into a peptide profile (described in Section 3), and selection of the precursor ions for MS/MS analysis were performed using an inhouse developed program written in Visual Basic 6 (Microsoft). Protein identification, both by peptide mass fingerprinting and fragment ion fingerprinting, was performed using the MASCOT software (Matrix Science, London, UK)
[19] using the Swiss-Prot (Release 41.3) and NCBI protein sequence databases. For protein identification, the following settings were used: mass error tolerance: $30 \mathrm{ppm}$; fixed modification: carbamidomethylation; variable modification: methionine oxidation; number of missed cleavages: one. For identification by fragment ion fingerprinting, additional parameters were: mass error tolerance for fragment ions: $0.8 \mathrm{Da}$; type of instrument: MALDI-TOF-PSD.

\section{Results and discussion}

\subsection{The design of the nano LC-MALDI interface}

Direct deposition of the LC effluent onto preformed microcrystalline matrix layers was found to yield highly reproducible spectra. The matrix layers were prepared prior to the nano LC experiment, by spreading the matrix solution over the surface of a prestructured sample support. This is a simple procedure by which a similar amount of matrix is deposited on each hydrophilic sample anchor. In addition, peptide standards, mixed into the matrix solution and prepared in this manner, yielded signals of even intensities, facilitating internal spectra calibration. At LC flow rates of $200-300 \mathrm{~nL} /$ min, each $20 \mathrm{~s}$ fraction has a volume of only $70-100 \mathrm{~nL}$. Because of the low volume of each deposited fraction, the matrix crystals were not dissolved, even at high percentages of organic solvent. No differences in spectra quality were observed between early and late fractions.

To ensure contact between the emerging liquid and the preformed matrix layers, the capillary outlet must be positioned close to the sample. A problem encountered, particu- 
larly at higher concentrations of organic solvent, was that the LC effluent showed a tendency to wet the outside of the capillary, with the consequence that not enough effluent remained below the capillary outlet to bridge the gap and thereby enable its deposition onto the sample support. For fused silica capillaries of $280 \mu \mathrm{m}$ od, this problem was observed even when the capillary outlet was positioned very close to the MALDI sample support $(40 \mu \mathrm{m})$. Using capillaries coated with Teflon did not eliminate this problem. Instead, using a capillary with a larger od (375 $\mu \mathrm{m}$ od) alleviated this problem by providing a sufficiently large crosssectional surface to hold the emerging droplet in place prior to its deposition onto the preformed matrix layers. The increase of the od from 280 to $375 \mu \mathrm{m}$ made the precision of the height positioning of the outlet of the capillary less critical. Thus, instead of $40 \mu \mathrm{m}$ above the surface for $280 \mu \mathrm{m}$ od capillaries, $140 \pm 10 \mu \mathrm{m}$ was used for $375 \mu \mathrm{m}$ od capillaries.

A gentle sheath-flow of nitrogen gas was used during fraction collection. Although not necessary for fraction collection, it increased the solvent evaporation rate, thereby decreasing the degree of methionine- and tryptophaneoxidation, which has previously been observed for this type of sample preparation [17]. Due to the faster evaporation rates at higher percentages of the organic solvent, the size of the droplet formed by the emerging liquid was smaller at the end of the gradient, requiring closer positioning of the capillary outlet above the MALDI support surface in comparison to the beginning of the gradient. Under these conditions, increasing the flow during the analytical separation was found to be advantageous. Ramping the flow rate from $300 \mathrm{~nL} / \mathrm{min}$ (at 30\% Buffer B) to $500 \mathrm{~nL} / \mathrm{min}$ (at $90 \%$ buffer $\mathrm{B})$, balanced the effect of increased evaporation rate at high percentages of organic solvent, and thereby improved the reliability of the fractionation.

As an alternative means to render the deposition of LC effluent more robust, we also evaluated the use of a liquid sheath-flow of acidic aqueous solution. A solution of $0.1 \%$ TFA was supplied through the port on the left side of the Tconnection (instead of nitrogen gas) by an isocratic LC pump at a flow rate of $2 \mu \mathrm{L} / \mathrm{min}$ and mixed with the LC effluent at the outlet of the LC capillary during deposition of the fraction onto the MALDI sample support. The volume of each $20 \mathrm{~s}$ fraction thus increased to $700 \mathrm{~nL}$, with the result that the accuracy of the distance between the capillary outlet and the sample support was less critical. The use of a liquid sheathflow, however, was found to have a serious drawback: it significantly increased the degree of oxidation of methionineand tryptophan-containing peptides. An example of this is shown in Fig. 2a. A similar effect was reported by Schuerenberg et al. [20] when preparing diluted samples on prestructured sample supports.

The presence of clusters of signals in the low molecular weight region was observed in some spectra. An example is shown in Fig. 3a. We could not correlate this problem, which affected approximately $5 \%$ of all samples, to any sample contaminant or impurities in the MALDI matrix. We found,

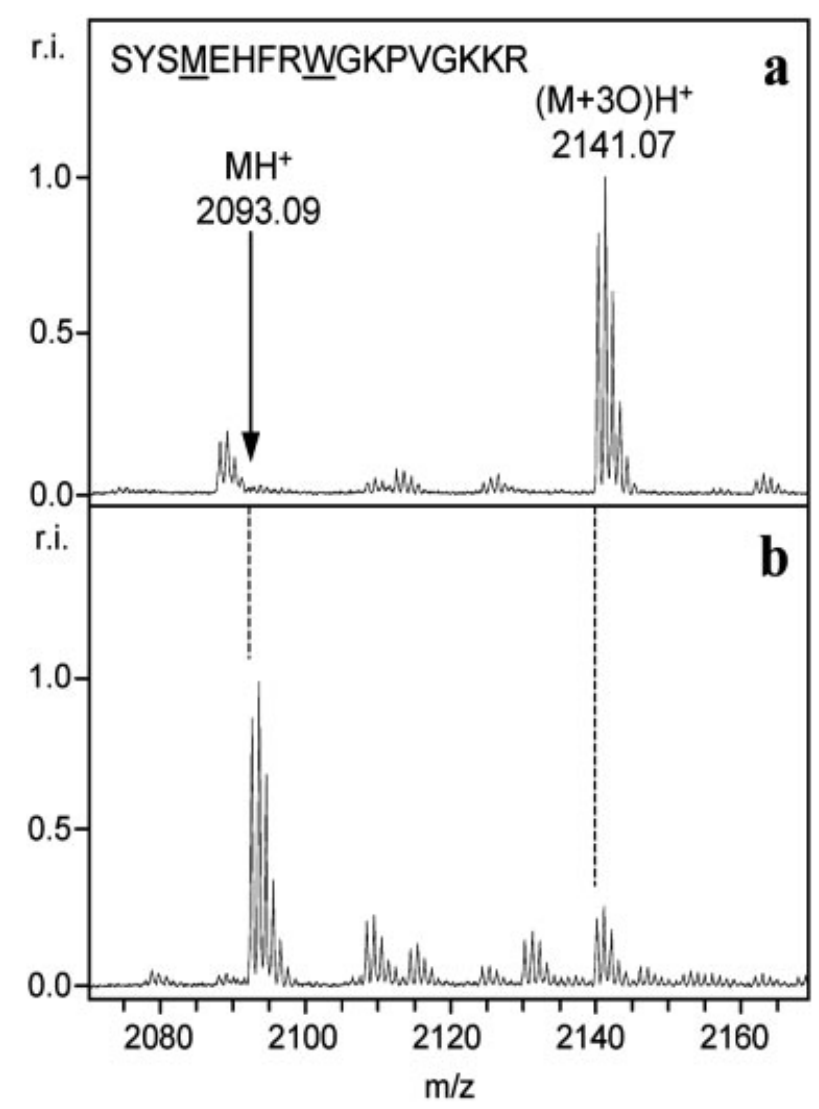

Figure 2. Comparison of fraction collection with and without assisting liquid sheath-flow. (a) Mass spectra of a peptide fractionated onto the MALDI sample plate using a liquid sheath-flow of $0.1 \%$ TFA. The presence of signal of high abundance at $\mathrm{m} / \mathrm{z}$ 2141.07 indicates nearly complete oxidation of the Met and Trp residues during MALDI sample preparation. (b) When the liquid sheath-flow was omitted, and instead a stream of nitrogen gas was directed onto the sample during fractionation, the degree of oxidation was decreased, with the signal of the highest abundance at $\mathrm{m} / \mathrm{z} 2093.09$, corresponding to the nonoxidized form of the peptide molecular ion.

however, that by including $50 \mathrm{~mm}$ citric acid in the MALDI matrix solution, this problem disappeared (Fig. 3b). The resulting spectra were markedly free from matrix-related signals in the low molecular weight region, improving the detection of peptides in the mass range below 800 Da. An example of this is shown in Fig. 3c.

\subsection{Data processing}

A general procedure was developed for processing and analyzing nano LC MALDI-MS data. In the following, the analysis of a tryptic digest of BSA (the amount analyzed corresponding to $50 \mathrm{fmol}$ of the digested protein) is used to illustrate the steps involved. Automatic peak detection yielded a total of 440 peaks in the 96 mass spectra. Figure 4a shows a plot of the fraction number (proportional to the elution time) of the detected signals versus their $m / z$ values. 


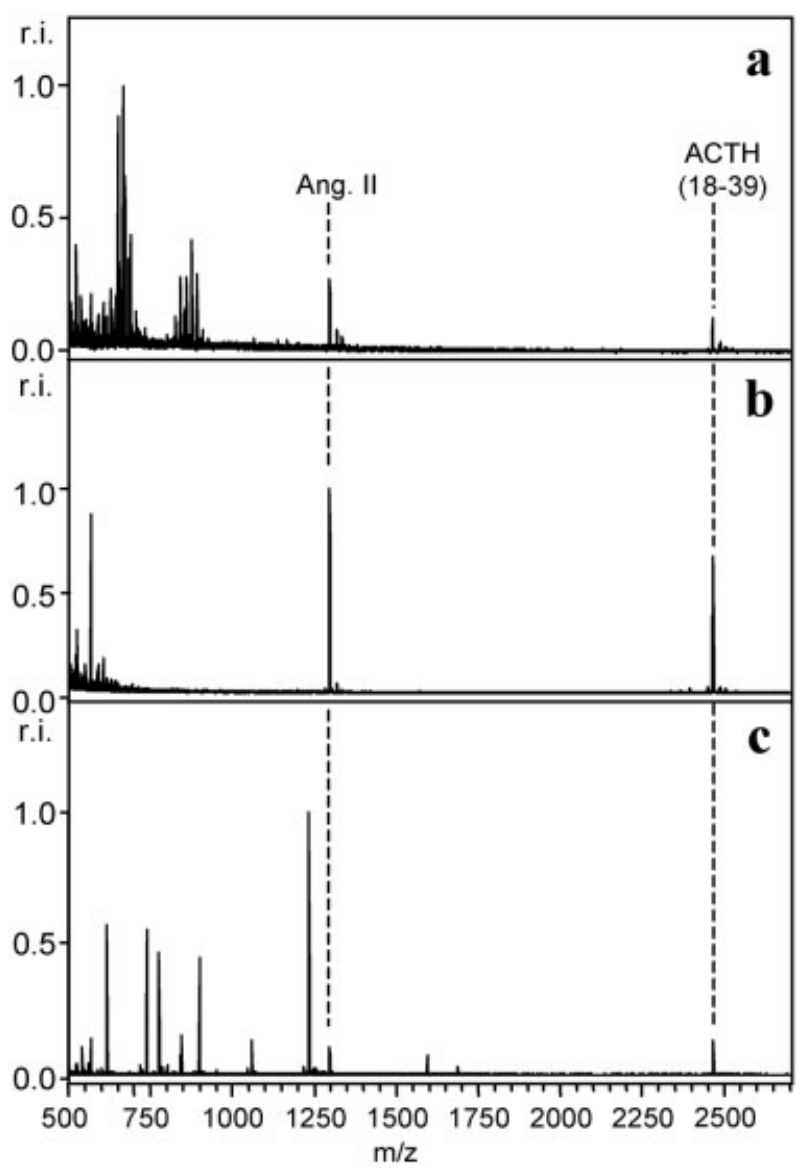

Figure 3. The effect of the addition of citric acid to the MALDI matrix solution. (a) MALDI-TOF mass spectrum of a blank LC fraction, containing only internal calibrants. In mass spectra acquired from approximately $5 \%$ of all fractions, strong signal clusters in the low mass range were observed. (b) When $50 \mathrm{~mm}$ citric acid was included in the matrix solution the clusters disappeared. (c) Mass spectrum of an LC fraction from a separation of a tryptic digest of $E$. coli $50 S$ ribosomal subunit. The inclusion of citric acid in the MALDI matrix solution improved the detection of peptides of low molecular weight.

\subsubsection{Peak filtering - adduct signals}

The acquired mass spectra contain not only signals that represent the protonated molecular ions of the sample molecules, but also various adduct ion signals and matrixderived signals, which obscure data interpretation. Frequently observed are sodium- and potassium-cationized molecular ions, appearing as satellite signals to the protonated peptide molecular ion signal with $\Delta m / z 21.982$ and 38.090 , respectively. Additionally, partial oxidation of methionine residues in peptides often occurs during the MALDI sample preparation, resulting in satellite signals with $\Delta m / z 15.994$. Filtering signals occurring within a mass window of $0.05 \mathrm{Da}$ around these values reduced the number of peaks to 429 .
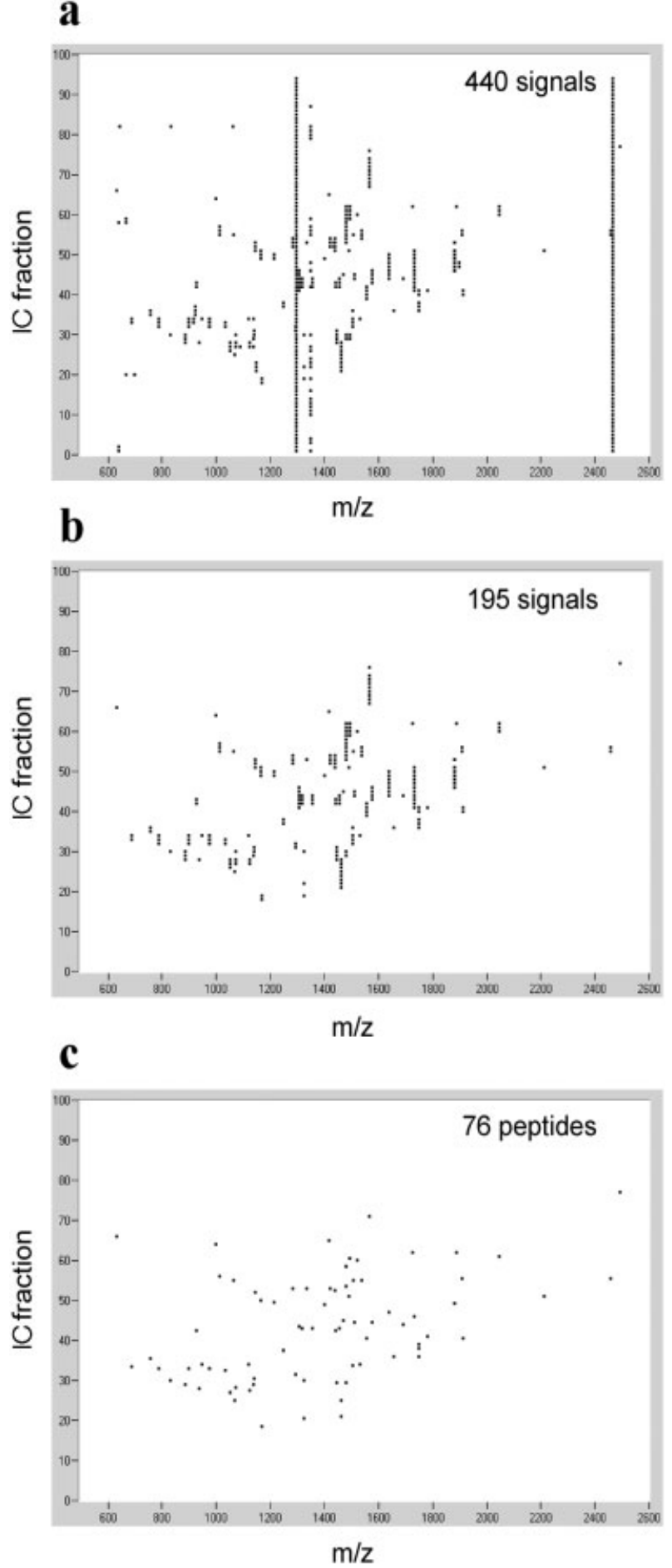

Figure 4. LC MALDI-MS Data processing. (a) The nano LC MALDITOF MS signal profile of a tryptic digest of BSA. In total, 440 monoisotopic signals were assigned in the entire set of mass spectra. (b) The signal profile after filtering of adduct signals, previously determined background signals, and signals of nonpeptide-like $m / z$ values: 195 signals remain after filtering. (c) The peptide profile of the sample, created by grouping the detected peptide ions according to their $\mathrm{m} / \mathrm{z}$ values and elution time, comprises 76 different sample components. 


\subsubsection{Peak filtering - non-peptidic signals}

In the region below $m / z$ 800, MALDI-TOF mass spectra often contain signals derived from the MALDI matrix or impurities therein. In some cases the compounds represented by these signals have elemental compositions that are sufficiently different from peptide elemental compositions that they may be distinguished from the latter by the decimal value of their molecular masses. The clustering of peptide molecular masses $[21,22]$ results in a window, for each unit mass below approximately $1500 \mathrm{Da}$, wherein no peptide molecular masses fall. The limits of this window was approximated according to the following:

A hypothetical unit mass (HUM) was calculated for Cys: the amino acid with the lowest decimal mass, and Leu/Ile: the amino acid with the highest decimal mass:

$$
\begin{aligned}
& \mathrm{HUM}_{\text {Cys }}=\mathrm{M}_{\text {Cys }} / \operatorname{Int}\left(\mathrm{M}_{\text {Cys }}\right)=103.0092 / 103=1.0000893204 \\
& \mathrm{HUM}_{\text {Leu }}=\mathrm{M}_{\text {Leu }} / \operatorname{Int}\left(\mathrm{M}_{\text {Leu }}\right)=113.0841 / 113=1.0007442478
\end{aligned}
$$

where $\mathrm{M}_{\mathrm{Cys}}$ and $\mathrm{M}_{\mathrm{Leu}}$ denote the monoisotopic molecular masses of Cys and Leu, respectively, and Int $\left(\mathrm{M}_{\mathrm{Cys}}\right)$ and Int $\left(\mathrm{M}_{\text {Leu }}\right)$ denote their corresponding nominal masses (integer values).

For each nominal mass (M) the minimum and maximum limits $\left(\mathrm{M}_{\min }\right.$ and $\left.\mathrm{M}_{\max }\right)$ of this window was calculated by:

$\mathrm{M}_{\min }=\mathrm{M} \times \mathrm{HUM}_{\mathrm{Cys}}$

$\mathrm{M}_{\max }=\mathrm{M} \times \mathrm{HUM}_{\mathrm{Leu}}$

Allowing for an error of $\pm 30 \mathrm{ppm}$, signals occurring outside this window were filtered out, resulting in 423 remaining signals in the current example (this approximation allows a peptide of any composition to have any nominal mass).

\subsubsection{Peak filtering - background signals}

Finally, the data set was filtered for signals occurring (within $\pm 30 \mathrm{ppm}$ ) in an exclusion mass list constructed from signals detected in a nano LC MALDI data set from a blank LC injection. Following this filtering, which removes signals derived from the MALDI matrix, the internal calibrants, and LC solvent system contaminants, 195 signals remained (Fig. 4b).

\subsubsection{Grouping of signals within a data set}

Because the same peptides are most often detected in several consecutive fractions, the filtered data set contains redundant information. For further analysis, the signals were grouped according to their $m / z$ value and fraction number (elution time). Signals detected in consecutive fractions with relative mass differences within $30 \mathrm{ppm}$ were grouped, and their average mass and elution time were calculated. Because weak signals can be suppressed in some fractions, and because of variations in data quality, a gap of two fractions was permitted. This grouping resulted in a peptide profile with 76 compounds, in which each peptide is characterized by an average molecular mass and elution time (Fig. 4c). Peptide profiles determined for different samples can be compared to each other to discern differences or similarities between samples.

\subsection{Evaluation of detection sensitivity}

A dilution series of a tryptic digest of BSA was prepared, with peptide concentrations corresponding to 50, 10, 5 and $1 \mathrm{fmol} /$ $\mu \mathrm{L}$ of the digested protein. Aliquots of $1 \mu \mathrm{L}$ were injected into the nano LC system (Agilent). Following calibration, filtering and grouping of the acquired mass spectrometric data, the number of peptides matching the BSA amino acid sequence was evaluated for each sample amount loaded. The results are summarized in Fig. 5 and Table 1. Approximately the same number of peptides was detected at 50 and $100 \mathrm{fmol}$ sample load. Below $50 \mathrm{fmol}$, the number of detected peptides decreased steeply with decreasing sample amount. A similar

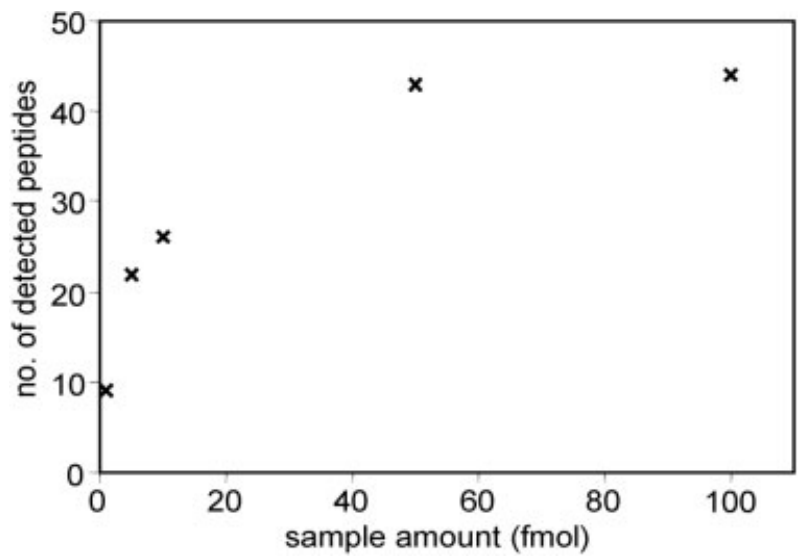

Figure 5. Detection sensitivity of nano LC MALDI-MS. A dilution series of a tryptic digest of BSA was analyzed, with peptide concentrations corresponding to $50,10,5$ and $1 \mathrm{fmol} / \mu \mathrm{L}$ of the digested protein. The number of detected peptides that matched the expected molecular masses of BSA tryptic peptides versus the loaded sample amounts are plotted.

Table 1. Detection sensitivity of nano LC MALDI-MS, determined by analysis of a dilution series of a tryptic digest of BSA. The number of matching peptide masses and sequence coverage at each analyte amount is listed

\begin{tabular}{lll}
\hline $\begin{array}{l}\text { Analyte amount } \\
\text { (fmol) }\end{array}$ & $\begin{array}{l}\text { No. of matched } \\
\text { peptides }\end{array}$ & $\begin{array}{l}\text { Sequence coverage } \\
(\%)\end{array}$ \\
\hline 100 & 44 & 72 \\
50 & 43 & 72 \\
10 & 26 & 46 \\
5 & 22 & 40 \\
1 & 9 & 20 \\
\hline
\end{tabular}


trend has been observed previously [17] for direct MALDITOF MS analysis of dilutions of peptide mixtures, and may mostly reflect losses of analyte molecules by adsorption to the sample vials and other surfaces. At the lowest loaded sample amount, 1 fmol, nine BSA tryptic peptides were detected, covering $20 \%$ of the amino acid sequence of the protein.

\subsection{Analysis of proteins isolated by gel electrophoresis}

Six proteins, isolated by 2-DE from a total protein extract of mouse brain that could not be identified by direct MALDI-MS peptide mass fingerprint analysis, or that had a probabilitybased MOWSE score [23] just above the significance threshold, were analyzed by nano LC MALDI-MS (using the Agilent LC). For both direct MALDI-MS and nano LC MALDI-MS analyses, the number of matching peptides and the score for each protein are given in Table 2 . The identity of each protein was confirmed by MS/MS fragment ion fingerprinting (data not shown). In all six examples, the number of detected peptides was higher for nano LC MALDI-MS compared to direct MALDI-MS analysis, with the consequence that the MOWSE score for protein identification was also higher.

Table 2. Nano LC MALDI-MS compared to direct MALDI-MS mixture analysis for protein identification by PMF. Six mouse brain proteins, isolated by 2-DE, that could not be identified by direct MALDI-MS analysis, or that had probability-based MOWSE scores just above the significance threshold of 65, were analyzed by nano LC MALDI-MS. For both analyses, the number of matching peptides, sequence coverage, and the scores for each protein are given in the table. The identity of each protein was confirmed by MS/MS

\begin{tabular}{llllll}
\hline \multicolumn{3}{c}{$\begin{array}{c}\text { Matching peptides/seq. } \\
\text { coverage }(\%)\end{array}$} & & \multicolumn{2}{c}{ MOWSE score } \\
\cline { 1 - 2 } \cline { 5 - 6 } NCBI GI & Direct & LC & & Direct & LC \\
no. & MALDI MS & MALDI-MS & & MALDI MS & MALDI-MS \\
\hline 6753496 & $7 / 14$ & $14 / 24$ & & 65 & 110 \\
18702315 & $9 / 12$ & $13 / 15$ & & 54 & 66 \\
14250287 & $12 / 16$ & $14 / 19$ & & 62 & 85 \\
3046247 & $4 / 14$ & $11 / 29$ & & 53 & 105 \\
4506127 & $5 / 19$ & $11 / 25$ & & 69 & 84 \\
18079339 & $5 / 9$ & $9 / 12$ & & 36 & 67 \\
\cline { 1 - 1 } & & & &
\end{tabular}

\subsection{Identification of proteins in mixtures}

The ability to identify proteins in mixtures was evaluated by the analysis of the E. coli 50S ribosomal subunit, known to consist of 33 different proteins [24]. A tryptic digest of the isolated protein complex was analyzed by nano LC MALDITOF MS using the LC Packings system. A total of 1934 signals were detected in the data set. The peptide profile, obtained after peak filtering and grouping of signals as described in Section 3.4, comprised 616 peptides (Fig. 6a);
235 signals with absolute intensities $>700$ counts and no adjacent interfering signals $( \pm 10 \mathrm{Da}$ ), corresponding to 216 different peptides (according to the signal grouping described above), were automatically selected for MALDI-TOF/ TOF fragment ion analysis. The 235 acquired fragment mass fingerprints were searched against all $E$. coli protein sequences in the Swiss-Prot database. Out of the submitted peak lists, $121(51 \%)$ yielded a significant $(p<0.05)$ MOWSE score and covered 30 of the expected 33 constituent proteins of the 50S subunit. These results are summarized in Table 3. Out of these 30, 24 were identified based on the database search results of three fragmented peptides, four based on two fragmented peptides, and two based on only a single fragment ion fingerprint. The three expected proteins (L23, L35, L36) that were not identified all have low molecular masses (11.2, 7.2, and $4.5 \mathrm{kDa}$, respectively). Upon complete cleavage with trypsin, both L35 and L36 are expected to produce only a single peptide each with a molecular mass $>500 \mathrm{Da}$, which might explain why they were not detected.

Table 3. The identification results of nano LC MALDI-MS analysis of $E$. coli 50 S ribosomal subunit

\begin{tabular}{|c|c|c|c|}
\hline Protein & Mass (kDa) & $\begin{array}{l}\text { No. of identi- } \\
\text { fied peptides }\end{array}$ & $\begin{array}{l}\text { Sequence } \\
\text { coverage }(\%)\end{array}$ \\
\hline L1 & 24.6 & 3 & 16 \\
\hline L2 & 29.7 & 8 & 30 \\
\hline L3 & 22.2 & 5 & 27 \\
\hline L4 & 22.1 & 5 & 33 \\
\hline L5 & 20.2 & 6 & 24 \\
\hline L6 & 18.8 & 6 & 34 \\
\hline L7/L12 & 12.1 & 5 & 43 \\
\hline L9 & 15.8 & 6 & 33 \\
\hline L10 & 17.6 & 5 & 31 \\
\hline L11 & 14.8 & 1 & 7 \\
\hline L13 & 16.0 & 5 & 31 \\
\hline L14 & 13.7 & 4 & 30 \\
\hline L15 & 14.9 & 4 & 26 \\
\hline L16 & 15.3 & 3 & 38 \\
\hline L17 & 14.4 & 5 & 36 \\
\hline L18 & 12.8 & 7 & 38 \\
\hline L19 & 13.0 & 5 & 57 \\
\hline L20 & 13.4 & 6 & 33 \\
\hline L21 & 11.6 & 2 & 19 \\
\hline L22 & 12.2 & 3 & 31 \\
\hline L23 & 11.2 & 0 & - \\
\hline L24 & 11.2 & 4 & 48 \\
\hline L25 & 10.7 & 2 & 22 \\
\hline L27 & 9.1 & 3 & 38 \\
\hline L28 & 8.9 & 3 & 42 \\
\hline L29 & 7.3 & 2 & 33 \\
\hline L30 & 6.4 & 5 & 59 \\
\hline L31 & 8.1 & 4 & 53 \\
\hline L32 & 6.3 & 2 & 45 \\
\hline L33 & 6.2 & 3 & 52 \\
\hline L34 & 5.4 & 1 & 17 \\
\hline L35 & 7.2 & 0 & - \\
\hline L36 & 4.5 & 0 & - \\
\hline
\end{tabular}




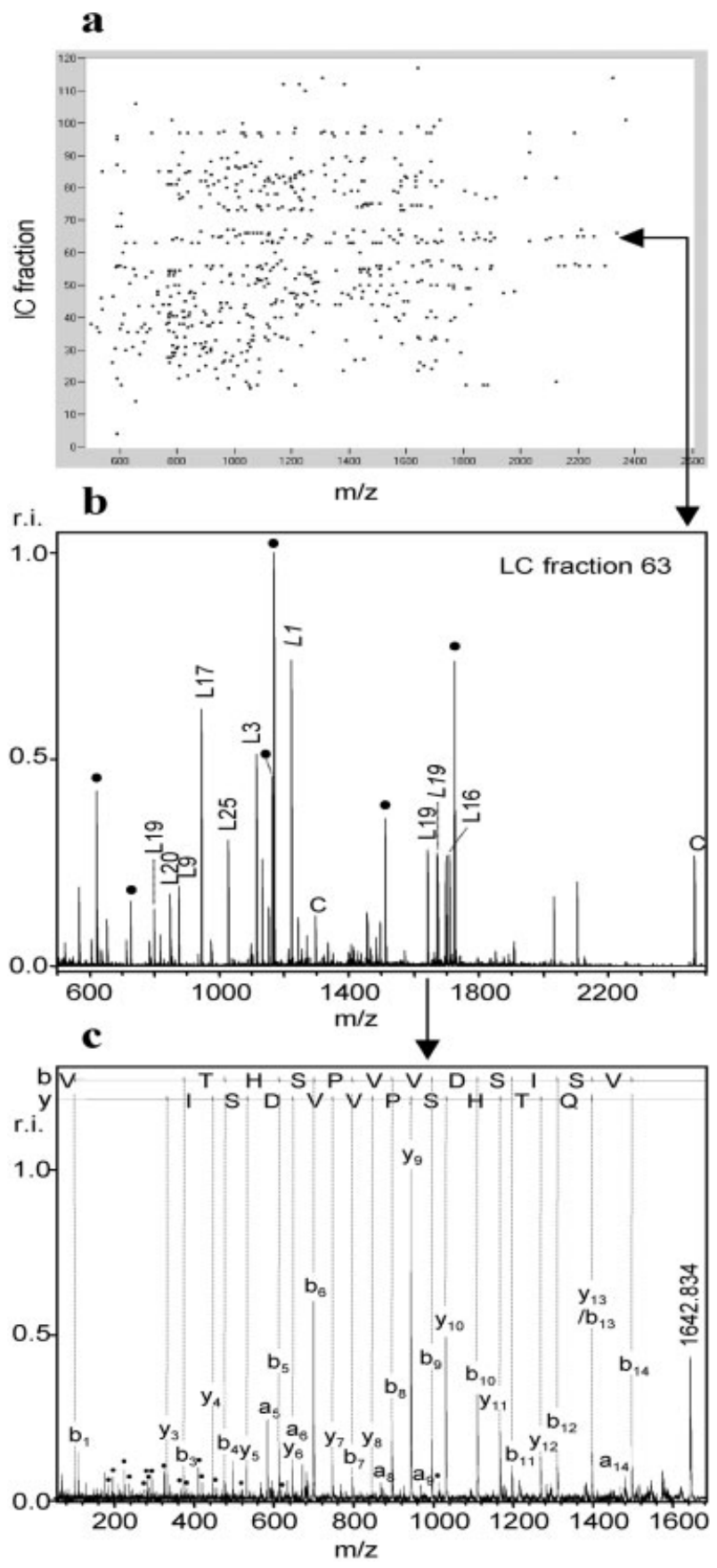

Figure 6. Identification of proteins in mixtures. (a) Nano LC MALDI-TOF MS peptide profile of $E$. coli $50 S$ ribosomal subunit. (b) MALDI-TOF mass spectrum of fraction 63. Peptides identified by database search are labeled with their corresponding protein names. Signals marked with a dot yielded fragment ion spectra of poor quality. Signals marked in italics were assigned based on fragment ion spectra acquired from neighboring fractions. (c) MALDI MS/MS spectrum of the peptide ion of $\mathrm{m} / \mathrm{z} 1642.834$. A fragment ion fingerprint database search identified the tryptic peptide VFOTHSPVVDSISVK, from the $50 \mathrm{~S}$ ribosomal protein L19. The $b$ - and $y$-ion series are assigned in the spectrum. A number of weaker signals that matched internal ya- and yb-fragments are indicated with dots.
The ability to store the separated peptides on the MALDI sample support has several advantages. Figure 6b shows the mass spectrum of fraction 63, which contains several co-eluting peptides. When using an on-line LC-ESI MS interface, such situations often leave insufficient time for isolation and acquisition of fragment ion spectra of all peptides of interest. For off-line LC MALDI-MS, however, the time available for MS/MS analysis is practically unlimited. In total, 14 fragment ion spectra of different peptides were acquired from fraction 63, yielding significant identification results in eight cases. Figure $6 c$ shows an example of the fragment ion spectrum acquired from the peptide of $m / z$ 1642.83. While spectra acquisition in MS mode consumes a very small portion of the MALDI sample, the acquisition of MALDI MS/MS spectra, however, results in ablation of a significant part of it. The miniaturized sample preparations used in this study (600 $\mu \mathrm{m}$ sample diameter) typically allowed the acquisition of 15-20 fragment ion spectra (each the sum of 800-2000 single-shot spectra). If a larger number of peptides are present in one fraction, this however, does not usually pose a problem because some of them are also present in neighboring fractions, in which they can be analyzed instead. For example, the two peptides of $\mathrm{m} / z 1222.72$ and 1673.78 , indicated in italics in Fig. 6b, were analyzed in MS/MS mode in neighboring fractions, enabling us to analyze all peptides of interest in fraction 63.

Another benefit attributable to the off-line nature of the interface is that it allows evaluation of the entire MS data set prior to selecting precursor ions and performing MS/MS analyses. Thus, the selection of precursor ions for fragmentation can be optimized based on the analysis of all MS data. Furthermore, the selection of precursor ions can be updated as new MS/MS data is being acquired. For example, when the fragment ion spectrum of a given peptide leads to a significant identification result for a protein, the molecular masses of all expected tryptic cleavage products from that protein can be calculated and matched to the experimentally determined masses of peptides in the dataset. After acquisition of MS/MS spectra of one or two of these peptides for confirmation of the identification, the remaining matching peptides can be filtered out or placed low on the priority list for MS/MS analysis, since they are likely to be derived from the same protein. As a result, it is possible to acquire sets of MS/MS data with low redundancy and high usable information content. In the current example, more than $50 \%$ of the acquired spectra led to significant identification results and most of the proteins were identified based on the search results from two or three fragment ion spectra.

\section{Concluding remarks}

We have presented a simple and robust off-line nano LC MALDI interface, based on deposition of LC effluent by adhesion of the emerging LC effluent to preformed MALDI matrix spots. The fractionation could be performed fully 
unattended for several LC separations. The deposited fractions resulted in homogeneous and reproducible MALDI sample preparations, well suited for automatic mass analysis. The nano LC MALDI interface described allowed the detection of peptides in samples containing low femtomole amounts. For proteins isolated by 2-DE, nano LC MALDI-MS resulted in the detection of more peptides compared to direct MALDI-TOF MS peptide mass fingerprinting due to reduced ion suppression, thereby improving the identification results. In combination with MALDI MS/MS analysis, the technique is powerful for the identification of proteins in mixtures, as shown here for the analysis of the E. coli $50 \mathrm{~S}$ ribosomal subunit.

While LC-ESI MS has undergone several years of development and, at present, is a well established and automated technique in many laboratories, this is not yet the case for LC MALDI-MS. Data acquisition takes a longer time for LC MALDI-MS and, in many cases, operator-controlled acquisition yield spectra of higher quality than those obtained acquired using the available automatic acquisition software. However, these limitations of LC MALDI-MS are not fundamental. The increased repetition rate of desorption/ionization lasers (up to the $\mathrm{kHz}$ range), faster data acquisition systems, and improved software for automatic data processing and acquisition control, is likely to bring the technique to a similar degree of automation as LC-ESI MS, and allow researchers to take full advantage of the benefits of an off-line interface.

The authors thank N. Gustavsson, K.-D. Kloeppel, and D. Weichart at the Max-Planck Institute for Molecular Genetics, Berlin, and Eckhard Nordhoff for scientific discussion. Daniel Wilson (Max-Planck Institute for Molecular Genetics, Berlin) is kindly acknowledged for assistance with preparation of the ribosome samples, and Martin Müller for programming the xyz-stage control software. This work was funded by the German Ministry for Education and Research (BMBF project 31P2715), and the Max-Planck Society.

\section{References}

[1] Gaskell, S. J., J. Mass Spectrom. 1997, 32, 677-688.

[2] Mehlis, B., Kertscher, U., Anal. Chim. Acta 1997, 352, 71-83.

[3] Karas, M., Hillenkamp, F., Anal. Chem. 1988, 60, 2301-2303.

[4] Murray, K. K., Mass Spectrom. Rev. 1997, 16, 283-299.

[5] Medzihradszky, K. F., Campbell, J. M., Baldwin, M. A., Falick, A. M. et al., Anal. Chem. 2000, 72, 552-558.

[6] Suckau, D., Resemann, A., Schuerenberg, M., Hufnagel, P. et al., Anal. Bioanal. Chem. 2003, 376, 952-965.

[7] Miliotis, T., Kjellstrom, S., Nilsson, J., Laurell, T. et al., J. Mass Spectrom. 2000, 35, 369-377.

[8] Ericson, C., Phung, Q. T., Horn, D. M., Peters, E. C. et al., Anal. Chem. 2003, 75, 2309-2315.

[9] Rejtar, T., Hu, P., Juhasz, P., Campbell, J. M. et al., J. Proteome Res. 2002, 1, 171-179.

[10] Wall, D. B., Berger, S. J., Finch, J. W., Cohen, S. A. et al., Electrophoresis 2002, 23, 3193-3204.

[11] Preisler, J., Foret, F., Karger, B. L., Anal. Chem. 1998, 70, 5278-5287.

[12] Zhan, Q., Gusev, A., Hercules, D. M., Rapid Commun. Mass Spectrom. 1999, 13, 2278-2283.

[13] Orsnes, H., Graf, T., Degn, H., Murray, K. K., Anal. Chem. 2000, 72, 251-254

[14] Bommer, U., Burkhardt, N., Jünemann, R., Spahn, C. M. T. et al., in: Graham, J., Rickwoods, D. (Eds.), Subcellular Fractionation. A Practical Approach, IRL Press, Oxford 1997, pp. 271-301.

[15] Spedding, G., in: Spedding, G. (Ed.) Ribosomes and Protein Synthesis: A Practical Approach, IRL Press, Oxford 1990, pp. 5-6.

[16] Shevchenko, A., Wilm, M., Vorm, O., Mann, M., Anal. Chem. $1996,68,850-858$.

[17] Gobom, J., Schuerenberg, M., Mueller, M., Theiss, D. et al., Anal. Chem. 2001, 73, 434-438.

[18] Gobom, J., Mueller, M., Egelhofer, V., Theiss, D. et al., Anal. Chem. 2002, 74, 3915-3923.

[19] Perkins, D. N., Pappin, D. J. C., Creasy, D. M., Cottrell, J. S., Electrophoresis 1999, 20, 3551-3567.

[20] Schuerenberg, M., Luebbert, C., Eickhoff, H., Kalkum, M. et al., Anal. Chem. 2000, 72, 3436-3442.

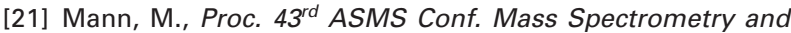
Allied Topics, Atlanta, GA, 1995, p. 639.

[22] Gay, S., Binz, P. A., Hochstrasser, D. F., Appel, R. D., Electrophoresis 1999, 20, 3527-3534.

[23] Pappin, D. J. C., Hojrup, P., Bleasby, A. J., Curr. Biol. 1993, 3, 327-332.

[24] Wittmann-Liebold, B., in: Hardesty, B., Kramer, G. (Eds.), Structure, Function and Genetics of Ribosomes, Springer, Berlin, Heidelberg, New York 1986, pp. 326-361. 